Original Article

\title{
Muscle activity of the vastus medialis obliquus during squat motion after static stretching of the tensor fasciae latae
}

\author{
Yuki Ohyama, RPT, MS $\left.{ }^{1,2}\right)^{*}$, TAkehiko Yamaji, RPT, PhD ${ }^{1)}$, Ena SAto, RPT, $\mathrm{PhD}^{1)}$, \\ Hideomi Watanabe, MD, $\mathrm{PhD}^{1)}$ \\ 1) Graduate School of Health Sciences, Gunma University, Japan \\ 2) Rehabilitation Center, Hidaka Rehabilitation Hospital: 2204 Maniwa Yoshii-machi, Takasaki-shi, \\ Gunma 370-2104, Japan
}

\begin{abstract}
Purpose] The purpose of this study was to investigate the changes of muscle activity during squat motion after stretching the tensor fasciae latae muscle. [Participants and Methods] The participants comprised 19 healthy males. The test muscles were the vastus medialis obliquus and vastus lateralis. The participants performed the squat motion before and after stretching the tensor fasciae latae. We measured the muscle activities and the onset timing of the vastus medialis obliquus relative to that of the vastus lateralis during squat motions. [Results] The onset timing of the vastus medialis obliquus relative to that of the vastus lateralis was decreased, and the vastus medialis obliquus activity and vastus medialis obliquus/vastus lateralis activity ratio were increased after stretching the tensor fasciae latae. [Conclusion] This study revealed that stretching of the tensor fasciae latae increases the relative vastus medialis obliquus muscle activity.

Key words: Vastus medialis obliquus, Squat motion, Surface electromyography
\end{abstract}

(This article was submitted Nov. 5, 2019, and was accepted Dec. 9, 2019)

\section{INTRODUCTION}

Preventing knee pathologies is very important in rehabilitation. One of the cause of knee pathologies is considered to be maltracking of the patella due to weak activity of the vastus medialis obliquus (VMO) compared to the vastus lateralis $(\mathrm{VL})^{1,2)}$. The relative activation of these muscles can be measured using electromyography (EMG). VMO/VL amplitude ratio $(\mathrm{VMO} / \mathrm{VL})$ and onset timing of $\mathrm{VMO}$ relative to $\mathrm{VL}(\mathrm{VMO}-\mathrm{VL})$ have been used as indicators of the relationship between these two muscles ${ }^{3,4)}$.

Closed-kinetic chain exercises, such as squat motions, are commonly recommended for preventing knee pathologies, as such exercises supposedly promote functional stability ${ }^{5}$, and have often been used as a variable motion in previous studies of $\mathrm{VMO} / \mathrm{VL}$ and $\mathrm{VMO}-\mathrm{VL}^{1,6)}$. As a method of squat motion, examination of VMO/VL according to differences in the position of the lower extremity has been performed ${ }^{1,3}$. Changes in relative VMO muscle activity may also be caused by changes in muscle flexibility, as well as changes in position. On the other hand, the tensor fasciae latae muscle (TFL) is arrested at the iliotibial band, and part of the iliotibial band is arrested at the patella, so tightness of the TFL appears likely to be involved in lateral displacement of the patella ${ }^{7-9}$. However, whether flexibility of the TFL is involved in maltracking of the patella and changes in VMO/VL and VMO-VL remains uncertain. The purpose of the present study was therefore to investigate the influence on muscle activity of the VMO and VL during squat motion after static stretching (SS) of the TFL.

*Corresponding author. Yuki Ohyama (E-mail: h172c001@gunma-u.ac.jp)

(C2020 The Society of Physical Therapy Science. Published by IPEC Inc.

(c) (1) $($ This is an open-access article distributed under the terms of the Creative Commons Attribution Non-Commercial No Derivatives cc) 


\section{PARTICIPANTS AND METHODS}

Participants were 19 healthy males (mean \pm SD: mean age, $24.3 \pm 1.3$ years; mean height, $171.1 \pm 5.4 \mathrm{~cm}$; mean weight, $65.3 \pm 9.2 \mathrm{~kg}$ ). Participants had no history of injury or physical functional impairment, and no current knee pain. All participants provided informed consent before participating in the present study. All procedures followed were in accordance with the ethical standards of the responsible committee on human experimentation (institutional and national) and with the Helsinki Declaration of 1964 and later revisions. This study was approved by the research ethics committee at Hidaka Rehabilitation Hospital (permission number: 190201).

SS of TFL was conducted according to the method described by Umehara and Ikezoe ${ }^{10)}$. Briefly, each participant was positioned supine on a bed with the trunk securely fixed by a non-elastic band. The right lower limb was chosen for measurement. The lower limb of the non-measured side (left side) was held in $125^{\circ}$ of hip flexion and maximal knee flexion to maintain posterior pelvic tilt. For stretching positions, the hip was kept in maximal adduction and maximal extension and the knee in $90^{\circ}$ flexion. SS for $60 \mathrm{~s}$ was carried out five times in total, with a 10-s interval between stretches.

Participants performed the squat motion three times before and after SS. They stood with their arms folded lightly and feet spread to shoulder width (resting position). The squat motion consisted of a 3-s descent, a static contraction for $5 \mathrm{~s}$ (squat position) and finally a 3 -s ascent ${ }^{1}$. After SS, squat motion were performed after a 10-second break. Angles of bilateral joints during the squat position were: hip joint, $80^{\circ}$ flexion; knee joint, $80^{\circ}$ flexion; ankle joint, $25^{\circ}$ dorsiflexion. While the squat position was maintained, the measurer measured each joint angle using a goniometer and checked whether it was held at the prescribed angle.

Rectus femoris (RF), VMO and VL activities of the right lower extremity were measured using EMG (WEB-7000; Nihon Kohden, Tokyo, Japan). Electrodes for the RF were attached at the midpoint along the line from the anterior spine iliac to the superior border of the patella ${ }^{11)}$. Electrodes for the VMO were attached $4 \mathrm{~cm}$ superior and $4 \mathrm{~cm}$ medial to the superomedial border of the patella at approximately $55^{\circ}$ from the long axis of the femur. Electrodes for the VL were attached $10 \mathrm{~cm}$ superior and 6-8 cm lateral to the superior lateral border of the patella and orientated $15^{\circ}$ from the vertical, as described previously ${ }^{2)}$. The raw signal was amplified and band-pass filtered $(15-500 \mathrm{~Hz})$, digitized at 1,000 Hz, and stored for offline analysis on the laboratory computer.

EMG data were full-wave rectified, and each average value of three measurements was taken as a representative value. Muscle onset time was determined as the point at which EMG amplitude increased to $>3$ SD from the baseline level (resting position) for a minimum of $25 \mathrm{~ms}^{4}$ ). EMG onsets identified by computer were visually confirmed. VMO-VL was obtained by subtracting the VL onset time from the VMO onset time. A negative value indicates that the VMO triggered earlier than $\mathrm{VL}$, whereas a positive value means that the VMO triggered later than the VL. The volume of muscle activity during the resting position and squat position for $3 \mathrm{~s}$ were standardized by maximal voluntary isometric contraction (MVC), and the calculated data were represented in the analysis as \%MVC. For MVC, participants were seated with their knee flexed at $90^{\circ}$ and resistance placed on the distal tibia. Verbal encouragement was given during MVC.

Statistical analyses were performed using SPSS Statistics for Windows version 22 (IBM, Tokyo, Japan). Values of the $\% \mathrm{MVC}$ of the two muscles and VMO/VL before and after SS were compared by paired t-tests. VMO-VL before and after SS were also compared by paired t-tests. Values of $\mathrm{p}<0.05$ were considered significant.

\section{RESULTS}

VMO-VL during squat motion was significantly smaller after SS than before SS (Table 1). This indicates that relative VMO muscle activity compared to VL may be earlier through the intervention with SS of the TFL.

VL activity in the resting position was significantly smaller after SS than before SS (Table 2). VMO activity and VMO/VL in the squat position were significantly larger after SS than before SS. The \%MVC of VMO was augmented by approximately $7 \%$ after SS. On the other hand, VL activity showed no significant difference before and after SS (Table 3). This indicates that the intervention of SS of the TFL may significantly strengthen muscle activity of VMO, but not VL.

Table 1. Difference in onset time (VMO-VL) during squat movements

\begin{tabular}{cc}
\hline Before SS $(\mathrm{ms})$ & After SS $(\mathrm{ms})$ \\
\hline $102.64 \pm 204.33$ & $5.89 \pm 197.26^{*}$ \\
\hline $\begin{array}{l}\text { Mean } \pm \text { standard deviation. } \\
{ }^{*} \mathrm{p}<0.05 .\end{array}$
\end{tabular}

Table 2. Volume of muscle with standing position

\begin{tabular}{lcc}
\hline & Before SS & \multicolumn{1}{c}{ After SS } \\
\hline RF (\%MVC) & $7.08 \pm 3.57$ & $6.88 \pm 3.36$ \\
VMO (\%MVC) & $2.72 \pm 1.49$ & $2.58 \pm 1.53$ \\
VL (\%MVC) & $6.19 \pm 3.60$ & $5.56 \pm 3.19^{*}$ \\
\hline
\end{tabular}

Mean \pm standard deviation.

$* \mathrm{p}<0.05$. 
Table 3. Volume of muscle with squat position

\begin{tabular}{lcc}
\hline & Before SS & After SS \\
\hline RF (\%MVC) & $21.46 \pm 13.08$ & $23.62 \pm 13.09$ \\
VMO (\%MVC) & $43.01 \pm 16.75$ & $46.20 \pm 17.27^{*}$ \\
VL (\%MVC) & $45.94 \pm 17.13$ & $45.81 \pm 16.46$ \\
VMO/VL (\%MVC) & $0.95 \pm 0.29$ & $1.03 \pm 0.31^{* *}$ \\
\hline
\end{tabular}

Mean \pm standard deviation.

$* \mathrm{p}<0.05 ; * \mathrm{p}<0.01$

\section{DISCUSSION}

In the present study, we measured muscle activity of both VMO and VL during squat motion before and after SS of the TFL in healthy adult men. As a result, after SS of the TFL, it became clear that the onset timing of VMO-VL decreased; that is, contraction of the VMO initiated immediately after initiation of VL contraction. In addition, VMO activity and VMO/VL during squat position increased.

First, VMO-VL at squat motion after SS of the TFL was significantly smaller than that before SS. TFL inserts into the iliotibial band, and part of the iliotibial band is considered to be involved in lateral displacement of the patella because it inserts into the patella ${ }^{7-9}$ ). With regard to movement of the patella, patella is located slightly outward during knee joint extension, and is displaced slightly inward at the initial stage of flexion ${ }^{12)}$. Lateral displacement of the patella is thus thought to be suppressed from the knee extension position to the initial stage of flexion after SS of TFL, and VMO activity involved in the inward displacement of the patella may be accelerated. In previous research involving healthy individuals, VMO-VL was assumed to increase in the group with knee joint pain after training when performing the same training for a fixed period, compared with before the training. Also, even before the start of training, the group with knee joint pain after training displayed significantly higher VMO-VL compared to the group without pain ${ }^{4}$. In healthy adults, accelerating relative onset of VMO contraction by SS of the TFL may lead to the prevention of the knee pathologies.

VL muscle activity was significantly decreased after SS of the TFL during the resting position. This result suggested that the TFL shows partial adhesion to the VL via the iliotibial band ${ }^{13)}$, and the VL may have been extended by SS of the TFL. On the other hand, VMO muscle activity was significantly increased after SS of the TFL in the squat position. VMO/VL ratio was also significantly increased after SS of the TFL. These results suggested that SS of the TFL reduced outward traction force on the patella, and VMO involved in the inward traction force of the patella became more active. That is, intervention with SS of the TFL may lead to augmentation of muscle activity of only the VMO specifically. To increase the dynamic stability of the patella, the $\mathrm{VMO}$ is required to contract more than the $\mathrm{VL}^{1}$. The resultant stability of the patella due to muscle elongation of the TFL is therefore suggested to affect the augmentation of VMO muscle activity, which may also contribute to patellar stability.

As one limitation of this study, quantitative measurement of patellar stability during squat motion was not evaluated. In the future, to consider the results of this study in terms of kinematics, it needs to be elucidated through the motion of the patella and three-dimensional motion analysis of the lower extremity during squat motion.

\section{Conflict of interest}

The authors have no financial or personal conflicts of interest to declare.

\section{REFERENCES}

1) Jaberzadeh S, Yeo D, Zoghi M: The effect of altering knee position and squat depth on VMO:VL EMG ratio during squat exercises. Physiother Res Int, 2016, 21: 164-173. [Medline] [CrossRef]

2) Coqueiro KR, Bevilaqua-Grossi D, Bérzin F, et al.: Analysis on the activation of the VMO and VLL muscles during semisquat exercises with and without hip adduction in individuals with patellofemoral pain syndrome. J Electromyogr Kinesiol, 2005, 15: 596-603. [Medline] [CrossRef]

3) Yoon JY, Kang MH, Oh JS: Effects of visual biofeedback using a laserbeam on the EMG ratio of the medial and lateral vasti muscles and kinematics of hip and knee joints during a squat exercise. J Phys Ther Sci, 2011, 23: 559-563. [CrossRef]

4) Van Tiggelen D, Cowan S, Coorevits P, et al.: Delayed vastus medialis obliquus to vastus lateralis onset timing contributes to the development of patellofemoral pain in previously healthy men: a prospective study. Am J Sports Med, 2009, 37: 1099-1105. [Medline] [CrossRef]

5) Irish SE, Millward AJ, Wride J, et al.: The effect of closed-kinetic chain exercises and open-kinetic chain exercise on the muscle activity of vastus medialis oblique and vastus lateralis. J Strength Cond Res, 2010, 24: 1256-1262. [Medline] [CrossRef]

6) Mostamand J, Bader DL, Hudson Z: Does leg predomination affect measuring vasti muscle onsets during single leg squatting? A reliability study. J Bodyw Mov Ther, 2017, 21: 763-766. [Medline] [CrossRef]

7) Kang SY, Choung SD, Park JH, et al.: The relationship between length of the iliotibial band and patellar position in Asians. Knee, 2014, 21: 1135-1138. [Medline] [CrossRef] 
8) Kwak SD, Ahmad CS, Gardner TR, et al.: Hamstrings and iliotibial band forces affect knee kinematics and contact pattern. J Orthop Res, 2000 , 18: 101-108. [Medline] [CrossRef]

9) Merican AM, Amis AA: Iliotibial band tension affects patellofemoral and tibiofemoral kinematics. J Biomech, 2009, 42: 1539-1546. [Medline] [CrossRef]

10) Umehara J, Ikezoe T, Nishishita S, et al.: Effect of hip and knee position on tensor fasciae latae elongation during stretching: an ultrasonic shear wave elastography study. Clin Biomech (Bristol, Avon), 2015, 30: 1056-1059. [Medline] [CrossRef]

11) Marchetti PH, Jarbas da Silva J, Jon Schoenfeld B, et al.: Muscle activation differs between three different knee joint-angle positions during a maximal Isometric Back Squat Exercise. J Sports Med Hindawi Publ Corp, 2016, 2016: 3846123. [Medline]

12) Grelsamer RP, Klein JR: The biomechanics of the patellofemoral joint. J Orthop Sports Phys Ther, 1998, 28: 286-298. [Medline] [CrossRef]

13) Merican AM, Amis AA: Anatomy of the lateral retinaculum of the knee. J Bone Joint Surg Br, 2008, 90: 527-534. [Medline] [CrossRef] 